\title{
Euler sums and non-integerness of harmonic type sums
}

\author{
Haydar Göral $^{1}$ (D), Doğa Can Sertbaş ${ }^{* 2}$ (1) \\ ${ }^{1}$ Department of Mathematics, Faculty of Sciences, Dokuz Eylül University, Tınaztepe Yerleşkesi, 35390 \\ Buca/İzmir, TURKEY \\ ${ }^{2}$ Department of Mathematics, Faculty of Sciences, Sivas Cumhuriyet University, 58140, Sivas, TURKEY
}

\begin{abstract}
We show that Euler sums of generalized hyperharmonic numbers can be evaluated in terms of Euler sums of generalized harmonic numbers and special values of the Riemann zeta function. Then we focus on the non-integerness of generalized hyperharmonic numbers. We prove that almost all generalized hyperharmonic numbers are not integers and our error term is sharp and the best possible. Finally, we analyze generalized hyperharmonic numbers in terms of topology and relate this to non-integerness.
\end{abstract}

Mathematics Subject Classification (2010). 11B83, 5A10, $11 \mathrm{~B} 75$

Keywords. Harmonic numbers, hyperharmonic numbers, Euler sums

\section{Introduction}

The goal of this paper is to study Euler sums and non-integerness of generalized hyperharmonic numbers. In order to achieve this, we make use of a recurrence relation of these numbers and the distribution of prime numbers. Before stating our results, we first give the necessary definitions. The sequence of partial sums of the harmonic series is called harmonic numbers, namely

$$
h_{n}=\sum_{k=1}^{n} \frac{1}{k}
$$

for $n \geq 1$. These numbers have been studied recurrently and exponentially. It is wellknown that

$$
h_{n}=\log n+\gamma+\mathcal{O}\left(\frac{1}{n}\right)
$$

and a finer one is

$$
\begin{aligned}
h_{n} & \sim \log n+\gamma+\frac{1}{2 n}-\sum_{k=1}^{\infty} \frac{B_{2 k}}{2 k n^{2 k}} \\
& =\log n+\gamma+\frac{1}{2 n}-\frac{1}{12 n^{2}}+\frac{1}{120 n^{4}}-\cdots
\end{aligned}
$$

\footnotetext{
*Corresponding Author.

Email addresses: hgoral@gmail.com (H. Göral), dogacan.sertbas@gmail.com (D.C. Sertbaş)

Received: 24.07.2018; Accepted: 30.01.2019
} 
as $n$ tends to infinity, where $\gamma$ is Euler's constant and $B_{m}$ is the $m$-th Bernoulli number. Recall that the Bernoulli numbers are defined as

$$
\frac{z}{e^{z}-1}=\sum_{n=1}^{\infty} \frac{B_{n}}{n !} z^{n}
$$

Linking the sum of the first $n$ positive integers with the sum of their reciprocals, that is to say with harmonic numbers, Ramanujan gave the striking asymptotic

$$
\begin{aligned}
h_{n} \sim \frac{1}{2} \log 2 m+\gamma & +\frac{1}{12 m}-\frac{1}{120 m^{2}}+\frac{1}{630 m^{3}}-\frac{1}{1680 m^{4}} \\
& +\frac{1}{2310 m^{5}}-\frac{191}{360360 m^{6}}+\frac{29}{30030 m^{7}} \\
& -\frac{2833}{1166880 m^{8}}+\frac{140051}{17459442 m^{9}}-\cdots
\end{aligned}
$$

as $n$ goes to infinity where

$$
m=\frac{n(n+1)}{2}=1+2+\cdots+n
$$

is the $n$-th triangular number. For the details, we refer the reader to page 531 of [6]. The $n$-th generalized harmonic number of order $m$, namely $H_{n}^{(m)}$, is defined as

$$
H_{n}^{(m)}=\sum_{k=1}^{n} \frac{1}{k^{m}}
$$

The generating function $\sum_{n=1}^{\infty} H_{n}^{(m)} z^{n}$ of $H_{n}^{(m)}$ is equal to

$$
\frac{\operatorname{Li}_{m}(z)}{1-z}
$$

where

$$
\operatorname{Li}_{m}(z)=\sum_{n=1}^{\infty} \frac{z^{n}}{n^{m}}
$$

is the polylogarithm function. Special values of the zeta function

$$
\mathrm{E}_{m}(s)=\sum_{k=1}^{\infty} \frac{H_{n}^{(m)}}{n^{s}}, \quad \Re(s)>1
$$

of $H_{n}^{(m)}$ are called Euler sums. In other words, for any integers $m \geq 1$ and $k \geq 2$, the sum $\mathrm{E}_{m}(k)$ is called an Euler sum. For $m=1$ and $k \geq 2$ an integer, Euler proved that

$$
\mathrm{E}_{1}(k)=\sum_{k=1}^{\infty} \frac{h_{n}}{n^{k}}=\frac{1}{2}(k+2) \zeta(k+1)-\frac{1}{2} \sum_{j=1}^{k-2} \zeta(k-j) \zeta(j+1),
$$

where

$$
\zeta(s)=\sum_{n=1}^{\infty} \frac{1}{n^{s}}
$$

is the Riemann zeta function defined in $\Re(s)>1$. Euler sums have received continuous attention and been reevaluated since then. For instance if $m+k$ is odd, then as in equation (1.6), the corresponding Euler sum can be evaluated in terms of the special values of the Riemann zeta function, see [7]. For more on the analytic properties of harmonic numbers and approximation type results, we refer the reader to [1]. Next, we define hyperharmonic numbers. Hyperharmonic numbers were first defined in the book of Conway and Guy 
[10] and they are another generalization of harmonic numbers. The n-th hyperharmonic number of order $r$ is defined recursively by

$$
h_{n}^{(r)}:=\sum_{k=1}^{n} h_{k}^{(r-1)}
$$

where $h_{n}^{(1)}=h_{n}$. By [10], one has that $h_{n}^{(r)}$ can be expressed in terms of binomial coefficients and harmonic numbers with the formula

$$
h_{n}^{(r)}=\left(\begin{array}{c}
n+r-1 \\
r-1
\end{array}\right)\left(h_{n+r-1}-h_{r-1}\right) .
$$

Mezö [15] conjectured that hyperharmonic numbers are never integers except 1, that is to say if $n \geq 2$ then $h_{n}^{(r)}$ cannot be an integer. The case $r=1$ was already proved by Theisinger [17]. Based on three different approaches, namely analytic, combinatorial and algebraic, the authors [11] proved that almost all hyperharmonic numbers are not integers. This yields an almost answer to Mező's problem [15]. Moreover, in the same paper [11], it was deduced that if $n$ is even or a prime power, or $r$ is odd then the corresponding hyperharmonic number is not integer.

Extending the definition of $h_{n}^{(r)}$ and $H_{n}^{(m)}$ simultaneously, generalized hyperharmonic numbers (see [9]) are defined by

$$
H_{n}^{(m, r)}:=\sum_{k=1}^{n} H_{k}^{(m, r-1)}, \quad r \geq 2,
$$

where $H_{n}^{(m, 1)}=H_{n}^{(m)}$ as defined by (1.5). In [9], Dirichlet series with generalized hyperharmonic numbers were computed in terms of the values of the Hurwitz zeta function. In the same paper, they also gave a combinatorial identity for generalized hyperharmonic numbers given by

$$
H_{n}^{(m, r)}=\sum_{j=1}^{n}\left(\begin{array}{c}
n-j+r-1 \\
r-1
\end{array}\right) \frac{1}{j^{m}}
$$

and this was generalized in [12, Proposition 2.1]. Unlike the hyperharmonic case $m=1$, we do not have such a formula as given in (1.8). Note also that we have

$$
\sum_{n=1}^{\infty} H_{n}^{(m, r)} z^{n}=\frac{\operatorname{Li}_{m}(z)}{(1-z)^{r}}
$$

Now we explain our results. Throughout the article, we let $n, m, r, k$ to be positive integers. Our first result states that Euler sums of generalized hyperharmonic numbers can be computed in terms of ordinary Euler sums and special values of the Riemann zeta function. For complex numbers $z_{1}, \ldots, z_{\ell}$, let $\left\langle z_{1}, \ldots, z_{\ell}\right\rangle_{\mathbb{Q}}$ denote the vector space generated by $z_{1}, \ldots, z_{\ell}$ over $\mathbb{Q}$. Euler sums of hyperharmonic numbers and analytic continuation of their Dirichlet series were studied in $[8,14]$. The next result is a structural theorem on Euler sums of generalized hyperharmonic numbers and generalizes the corresponding results of $[8,14]$.

Theorem 1.1. Let

$$
\zeta_{H^{(m, r)}}(s)=\sum_{n \geq 1} \frac{H_{n}^{(m, r)}}{n^{s}},
$$

where $m, r \geq 1$ and $\Re(s)>r$. For any integer $k \geq r+1$, the function $\zeta_{H^{(m, r)}}(k)$ can be written as a finite $\mathbb{Q}$-linear combinations of ordinary Euler sums and special values of the Riemann zeta function. Moreover, we have

$$
\zeta_{H^{(m, r)}}(k) \in\left\langle\mathrm{E}_{i}(j), \zeta(j): 1 \leq i \leq m, 2 \leq j \leq k\right\rangle_{\mathbb{Q}}
$$


and all rational coefficients are effectively computable in this expression. Furthermore, the function $\zeta_{H^{(m, r)}}(s)$ has a meromorphic continuation to the whole complex plane.

Some remarks are as follows: even in the case $r=2$ and $m+k$ is odd, it does not seem that the corresponding Euler sum $\zeta_{H^{(m, r)}}(k)$ can be expressed as a $\mathbb{Q}$-linear combinations of at most two products of special values of the Riemann zeta functions as in equation (1.6), see also [7]. Therefore the representation given in Theorem 1.1 seems to be the best possible one.

Our second theorem generalizes the result in [9] and it reveals the existence of plentiful non-integer generalized hyperharmonic numbers.

Theorem 1.2. Let $n, m, r$ be positive integers where $n \geq 2$.

(1) For any prime $p$ and $k \geq 1$, if $n=p^{k}$ then $H_{n}^{(m, r)}$ is not an integer.

(2) Let $p$ be the maximum prime that is less than $n$. If for all $c \in \mathbb{N}$, we have that $c p^{m} \notin[r, n-p+r-1]$, then $H_{n}^{(m, r)}$ is not an integer. In fact, for sufficiently large $n$, if $c p^{m} \notin\left[r, r+n^{0.525}-1\right]$ for all $c \in \mathbb{N}$, then $H_{n}^{(m, r)} \notin \mathbb{Z}$.

(3) If $n$ is sufficiently large and $r \leq\left(n-n^{0.525}\right)^{m}-n^{0.525}+1$, then $H_{n}^{(m, r)} \notin \mathbb{Z}$. In particular for a given $r$, if $n$ is large enough, then the corresponding $H_{n}^{(m, r)}$ is not an integer.

(4) If

$$
\frac{(n-1)}{(r-1) !} \prod_{i=-1}^{r-3}(n+i)<2^{m}
$$

holds, then $H_{n}^{(m, r)} \notin \mathbb{Z}$.

Let

$$
S(x)=\left|\left\{(n, r) \in[0, x] \times[0, x]: h_{n}^{(r)} \notin \mathbb{Z}\right\}\right| .
$$

In other words, $S(x)$ counts the number of pairs $(n, r)$ in the rectangle $[0, x] \times[0, x]$ where the corresponding hyperharmonic number $h_{n}^{(r)}$ is not an integer. In $[2,11]$, it was obtained that

$$
S(x) \sim x^{2},
$$

which means that non-integer hyperharmonics have the full asymptotic in the first quadruple. This result is based on primes in short intervals. Our third theorem extends this result to generalized hyperharmonic numbers.

\section{Theorem 1.3.}

(1) For sufficiently large $n$, the set of integers $r$ with $H_{n}^{(m, r)} \notin \mathbb{Z}$ contains a set of density

$$
1-\frac{n^{0.525}}{\left(n-n^{0.525}\right)^{m}}
$$

In particular, this density goes to 1 , if one of $n$ or $m$ tends to infinity.

(2) For a fixed $m \geq 2$, let

$$
S_{m}(x)=\left|\left\{(n, r) \in[0, x] \times[0, x] \mid H_{n}^{(m, r)} \notin \mathbb{Z}\right\}\right| .
$$

Then

$$
S_{m}(x)=x^{2}+\mathcal{O}_{m}\left(x^{1+\frac{1}{m}}\right) .
$$

In other words for a fixed $m \geq 2$, almost all generalized hyperharmonic numbers are not integers. 
Now we consider the case where $m$ is also not fixed. We obtain that almost all generalized hyperharmonic numbers are not integers as well. The following result is sharp and the best possible as for $n=1, H_{n}^{(m, r)}=1$ which is independent from the choice of $m$ and $r$. That is to say, the error term $\mathcal{O}\left(x^{2}\right)$ in the following theorem is inevitable and one cannot give a better error term.

Theorem 1.4. Let

$$
T(x)=\left|\left\{(n, m, r) \in[0, x]^{3} \mid H_{n}^{(m, r)} \notin \mathbb{Z}\right\}\right| .
$$

Then $T(x)=x^{3}+\mathcal{O}\left(x^{2}\right)$. This means that almost all generalized hyperharmonic numbers are not integers.

Next, we analyze generalized hyperharmonic numbers in terms of topology, and this leads to non-integerness of these numbers. Also the first part of the following theorem gives a characterization of Mező's problem [15].

\section{Theorem 1.5.}

(1) The set $\mathcal{H}=\left\{h_{n}^{(r)} \mid n, r \geq 1\right\}$ is closed in $\mathbb{R}$ in the usual topology. In particular, there exists an integer $a$ in $\mathcal{H}$ if and only if a is a limit point of the set $\mathcal{H}$.

(2) The topological closure of the set $\mathcal{G}=\left\{H_{n}^{(m, r)} \mid m, n, r \geq 1\right\}$ is

$$
\mathcal{G} \cup\{\zeta(k) \mid k \geq 2\} \cup\{1,2, \ldots\} .
$$

(3) Given $n, r \geq 1$, there exists $M=M(n, r)$ such that if $m \geq M$ then $H_{n}^{(m, r)} \notin \mathbb{Z}$.

Note that one can obtain Theorem 1.5 (3) from Theorem 1.2 (4) in an effective way. However, from topological point of view, this part follows immediately.

Now we fix our notations in this paper: we denote $p$ as a prime, unless it is stated otherwise. Also for a given $a \in \mathbb{Z}$, we define

$$
\nu_{p}(a):= \begin{cases}m & \text { if } p^{m} \| a \\ \infty & \text { if } a=0\end{cases}
$$

as the $p$-adic valuation of $a$. Here $p^{m} \| a$ means $p^{m} \mid a$ but $p^{m+1} \nmid a$. We extend this notation to a rational number $q=a / b \in \mathbb{Q}$ by $\nu_{p}(q)=\nu_{p}(a)-\nu_{p}(b)$ where $a, b \in \mathbb{Z}$. Note that for any $q_{1}, q_{2} \in \mathbb{Q}$, we have

$$
\begin{aligned}
\nu_{p}\left(q_{1} q_{2}\right) & =\nu_{p}\left(q_{1}\right)+\nu_{p}\left(q_{2}\right) \\
\nu_{p}\left(q_{1}+q_{2}\right) & \geq \min \left\{\nu_{p}\left(q_{2}\right), \nu_{p}\left(q_{2}\right)\right\},
\end{aligned}
$$

and the last property is called the non-Archimedian property of the p-adic valuation. Moreover we have equality in $(1.12)$ if $\nu_{p}\left(q_{1}\right) \neq \nu_{p}\left(q_{2}\right)$.

Next we define the big-O notation as given in [3]. Let $g(x)$ be a function from $\mathbb{R}$ to itself and suppose that $g(x)>0$ for $x \geq a$, where $a$ is a real number. We use the notation

$$
f(x)=\mathcal{O}(g(x))
$$

to mean that there exists a constant $c>0$ such that

$$
|f(x)| \leq c g(x), \text { for all } x \geq a .
$$

We write

$$
f(x)=\mathcal{O}_{\ell}(g(x))
$$

to indicate that the big-O constant may depend on $\ell$. We say that $f(x)$ is asymptotic to $g(x)$, denoted by $f(x) \sim g(x)$, if

$$
\lim _{x \rightarrow \infty} \frac{f(x)}{g(x)}=1 .
$$




\section{Proofs}

Proof of Theorem 1.1. By (1.11), we know that

$$
\sum_{n=1}^{\infty} H_{n}^{(m, r)} z^{n}=\frac{\operatorname{Li}_{m}(z)}{(1-z)^{r}}, \quad|z|<1 .
$$

Taking derivatives of both sides and using the equality

$$
\left(\operatorname{Li}_{m}(z)\right)^{\prime}=\frac{\operatorname{Li}_{m-1}(z)}{z},
$$

we get that

$$
\begin{aligned}
\sum_{n=1}^{\infty} n H_{n}^{(m, r)} z^{n-1} & =\frac{\frac{\operatorname{Li}_{m-1}(z)}{z}(1-z)^{r}+r(1-z)^{r-1} \operatorname{Li}_{m}(z)}{(1-z)^{2 r}} \\
& =\frac{\operatorname{Li}_{m-1}(z)}{z(1-z)^{r}}+\frac{r \operatorname{Li}_{m}(z)}{(1-z)^{r+1}}
\end{aligned}
$$

This yields by (1.11) again that

$$
\sum_{n=1}^{\infty} n H_{n}^{(m, r)} z^{n}=\sum_{n=1}^{\infty} H_{n}^{(m-1, r)} z^{n}+\sum_{n=1}^{\infty} r H_{n}^{(m, r+1)} z^{n+1} .
$$

By adjusting the index and comparing the coefficients, we obtain the following recurrence relation:

$$
r H_{n}^{(m, r+1)}=(n+1) H_{n+1}^{(m, r)}-H_{n+1}^{(m-1, r)} .
$$

To prove the first part of the theorem, we proceed by induction on $r$ and we apply equation (2.1). If $r=1$, then it is clear. Moreover if $m=1$ then the theorem follows from $[8,14]$. Now suppose the theorem for $1 \leq j \leq r$, and we will show it for $r+1$ where $m \geq 2$. By the recursion formula (1.9) for any $p \geq 1$, we know that

$$
\begin{aligned}
H_{n+1}^{(p, r)} & =H_{n}^{(p, r)}+H_{n+1}^{(p, r-1)} \\
& =H_{n}^{(p, r)}+\cdots+H_{n}^{(p, 2)}+H_{n}^{(p)}+\frac{1}{(n+1)^{p}} .
\end{aligned}
$$

By (2.1), we get that

$$
\begin{aligned}
r H_{n}^{(m, r+1)} & =(n+1) H_{n+1}^{(m, r)}-H_{n+1}^{(m-1, r)} \\
& =(n+1) \sum_{j=1}^{r} H_{n}^{(m, j)}-\sum_{j=1}^{r} H_{n}^{(m-1, j)} .
\end{aligned}
$$

So the corresponding zeta function can be found as

$$
\begin{aligned}
\zeta_{H^{(m, r+1)}}(s) & =\frac{1}{r}\left(\sum_{j=1}^{r} \sum_{n \geq 1} \frac{H_{n}^{(m, j)}}{n^{s-1}}+\sum_{j=1}^{r} \sum_{n \geq 1} \frac{H_{n}^{(m, j)}}{n^{s}}-\sum_{j=1}^{r} \sum_{n \geq 1} \frac{H_{n}^{(m-1, j)}}{n^{s}}\right) \\
& =\frac{1}{r} \sum_{j=1}^{r}\left(\zeta_{H^{(m, j)}}(s-1)+\zeta_{H^{(m, j)}}(s)-\zeta_{H^{(m-1, j)}}(s)\right) .
\end{aligned}
$$

By induction, we know that each of the summands on the right hand side of (2.2) can be written as a finite $\mathbb{Q}$-linear combinations of ordinary Euler sums and special values of the Riemann zeta function, when $s=k \geq r+1$. Moreover by equation (2.2) and induction on $r$, we deduce that

$$
\zeta_{H^{(m, r)}}(k) \in\left\langle\mathrm{E}_{i}(j), \zeta(j): 1 \leq i \leq m, 2 \leq j \leq k\right\rangle_{\mathbb{Q}} .
$$


For the last part of the theorem, when $r=1$, the meromorphic continuation follows from [4]. If $m=1$, then by [14] we finish the proof again. So suppose that $m, r \geq 2$. Now by induction on $r$ and the functional equation (2.2), we obtain the meromorphic continuation of $\zeta_{H^{(m, r)}}(s)$ to the whole complex plane.

Proof of Theorem 1.2 (1). Let $n=p^{k}$. By equation (1.10), we have

$$
H_{n}^{(m, r)}=\sum_{j=1}^{n}\left(\begin{array}{c}
n-j+r-1 \\
r-1
\end{array}\right) \frac{1}{j^{m}}=\frac{1}{p^{k m}}+\sum_{j=1}^{p^{k}-1}\left(\begin{array}{c}
p^{k}-j+r-1 \\
r-1
\end{array}\right) \frac{1}{j^{m}} .
$$

Note that $\nu_{p}(j)<k$, for any $j \in\{1, \ldots, n-1\}$. Therefore

$$
\nu_{p}\left(\left(\begin{array}{c}
p^{k}-j+r-1 \\
r-1
\end{array}\right) \frac{1}{j^{m}}\right) \geq(1-k) m \geq 1-k m .
$$

So by the non-Archimedian property of the $p$-adic valuation, we deduce that

$$
\nu_{p}\left(H_{n}^{(m, r)}\right)=\nu_{p}\left(p^{-k m}\right)=-k m .
$$

As $k, m \geq 1$, the non-integerness follows by the previous equation.

Proof of Theorem 1.2 (2). Let $p$ be the largest prime that is less than $n$. From the first part of the theorem, we may suppose that $n$ is not a prime. By the Bertrand's Postulate, we know that $\frac{n}{2}<p<n<2 p$. Therefore, we get

$$
H_{n}^{(m, r)}=\sum_{\substack{j=1 \\
j \neq p}}^{n}\left(\begin{array}{c}
n-j+r-1 \\
r-1
\end{array}\right) \frac{1}{j^{m}}+\left(\begin{array}{c}
n-p+r-1 \\
r-1
\end{array}\right) \frac{1}{p^{m}} .
$$

Notice that the first summand in equation (2.3) has a positive $p$-adic valuation as there is only one multiple of $p$ in $[1, n]$. Now we analyze the second summand. Observe that

$$
\left(\begin{array}{c}
n-p+r-1 \\
r-1
\end{array}\right)=\frac{r(r+1) \cdots(n-p+r-1)}{(n-p) !} .
$$

Note that there exists at most one multiple of $p$ in $[r, n-p+r-1]$, since the length of the corresponding interval is $n-p$ and $n-p<p$. As $c p^{m} \notin[r, n-p+r-1]$ for any $c \in \mathbb{N}$, we deduce that $p^{m} \nmid r(r+1) \cdots(n-p+r-1)$. Therefore

$$
\begin{aligned}
\nu_{p}\left(\left(\begin{array}{c}
n-p+r-1 \\
r-1
\end{array}\right)\right) & \leq \nu_{p}(r(r+1) \cdots(n-p+r-1)) \\
& \leq m-1 .
\end{aligned}
$$

Combining (2.3), (2.4) and the non-Archimedian property of the $p$-adic valuation, we get that $\nu_{p}\left(H_{n}^{(m, r)}\right) \leq-1$, which yields the non-integerness of $H_{n}^{(m, r)}$. Now suppose that $n$ is sufficiently large. By [5], we know that the prime $p$ lies in the interval $\left(n-n^{0.525}, n\right)$. Hence $n-p<n^{0.525}$, which implies that $[r, n-p+r-1] \subseteq\left[r, r+n^{0.525}-1\right]$. By the argument above, we see that if $c p^{m} \notin\left[r, r+n^{0.525}-1\right]$ for any $c \in \mathbb{N}$, then the non-integerness follows.

Proof of Theorem 1.2 (3). Suppose that $r \leq\left(n-n^{0.525}\right)^{m}-n^{0.525}+1$. Again we may assume by the first part of Theorem 1.2 that $n$ is not a prime. Since $n$ is sufficiently large, we obtain that the largest prime $p$ that is less than $n$ satisfies the inequality $n-n^{0.525}<$ $p<n$. Therefore $n-p<n^{0.525}$, which implies that

$$
r \leq\left(n-n^{0.525}\right)^{m}-n^{0.525}+1<p^{m}-(n-p)+1 .
$$


Observe that this inequality holds if and only if $n-p+r-1<p^{m}$. Note by this fact that $c p^{m} \notin[r, n-p+r-1]$ for any $c \in \mathbb{N}$, as $0<r \leq n-p+r-1<p^{m}$ and $1 \leq n-p$. By part (2) of Theorem 1.2, we conclude that $H_{n}^{(m, r)} \notin \mathbb{Z}$. The last statement follows directly.

Proof of Theorem 1.2 (4). Again by equation (1.10), we know that

$$
H_{n}^{(m, r)}=\left(\begin{array}{c}
n-1+r-1 \\
r-1
\end{array}\right)+\sum_{j=2}^{n}\left(\begin{array}{c}
n-j+r-1 \\
r-1
\end{array}\right) \frac{1}{j^{m}} .
$$

If the second summand in equation (2.6) is less than 1 , then $H_{n}^{(m, r)}$ cannot be integer. We may assume that $r \geq 2$, because if $r=1$ then non-integerness follows by [17] and the fact that

$$
1<H_{n}^{(m)}<\zeta(m) \leq \zeta(2)<2
$$

for $n, m \geq 2$. Suppose that the inequality

$$
\frac{(n-1)}{(r-1) !} \prod_{i=-1}^{r-3}(n+i)<2^{m}
$$

holds. Note that the product $(n-1) \cdots(n+r-3)$ is greater than or equal to the product $(n-j+1) \cdots(n-j+r-1)$ for any $j \geq 2$. Therefore, we have

$$
\sum_{j=2}^{n}\left(\begin{array}{c}
n-j+r-1 \\
r-1
\end{array}\right) \leq(n-1) \cdot\left(\begin{array}{c}
n-1+r-1 \\
r-1
\end{array}\right) .
$$

For $j \geq 2$, we see that

$$
\begin{aligned}
\sum_{j=2}^{n}\left(\begin{array}{c}
n-j+r-1 \\
r-1
\end{array}\right) \frac{1}{j^{m}} & \leq \frac{1}{2^{m}} \sum_{j=2}^{n}\left(\begin{array}{c}
n-j+r-1 \\
r-1
\end{array}\right) \\
& \leq \frac{n-1}{2^{m}} \cdot\left(\begin{array}{c}
n-1+r-1 \\
r-1
\end{array}\right) .
\end{aligned}
$$

By (2.7) and (2.8), we get that

$$
0<\sum_{j=2}^{n}\left(\begin{array}{c}
n-j+r-1 \\
r-1
\end{array}\right) \frac{1}{j^{m}}<1,
$$

and hence the non-integerness follows.

Proof of Theorem 1.3 (1). Let $n$ be sufficiently large, and $p$ denote the biggest prime that is less than $n$. Observe by Theorem $1.2(2)$ that if $c p^{m} \notin\left[r, r+n^{0.525}-1\right]$ for any $c \in \mathbb{N}$, then $H_{n}^{(m, r)} \notin \mathbb{Z}$. Thus the only possibility for $H_{n}^{(m, r)}$ to be an integer comes from the condition $r \in\left[c p^{m}-n^{0.525}+1, c p^{m}\right]$. When we consider the numbers modulo $p^{m}$, the observation above leads to the fact that the set of integers $r$ with $H_{n}^{(m, r)} \notin \mathbb{Z}$ contains a set of density

$$
1-\frac{n^{0.525}}{p^{m}}>1-\frac{n^{0.525}}{\left(n-n^{0.525}\right)^{m}}
$$

as $p>n-n^{0.525}$. This completes the proof.

Proof of Theorem 1.3 (2). Let $m \geq 2$ be fixed and put

$$
S_{m}(x)=\left|\left\{(n, r) \in[0, x] \times[0, x] \mid H_{n}^{(m, r)} \notin \mathbb{Z}\right\}\right| .
$$

By Theorem $1.2(3)$, we know that if $n$ is sufficiently large and $r \leq\left(n-n^{0.525}\right)^{m}-n^{0.525}+1$ then $H_{n}^{(m, r)} \notin \mathbb{Z}$. Note that $r=\mathcal{O}_{m}\left(n^{m}\right)$. Consider the following Figure 1. 


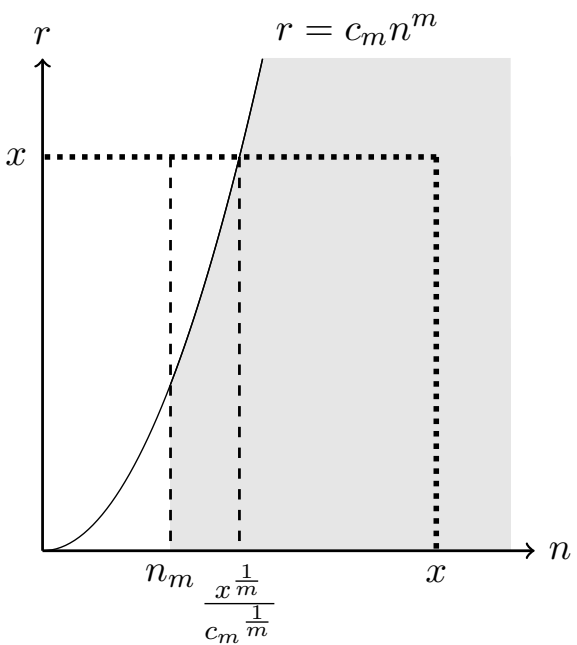

Figure 1. The graph of $r=c_{m} n^{m}$, for a fixed $m \geq 2$ and for some $c_{m}>0$. The shaded area indicates the lattice points $(n, r)$ where the corresponding generalized hyperharmonic number $H_{n}^{(m, r)}$ is not an integer.

Observe that the function intersects the line $r=x$ at $\left(\frac{x}{c_{m}}\right)^{\frac{1}{m}}$. Therefore there are at most $\mathcal{O}_{m}\left(x \cdot x^{\frac{1}{m}}\right)$ possibly many tuples $(n, r)$ where the corresponding generalized hyperharmonic $H_{n}^{(m, r)}$ may be an integer. Thus we get that $S_{m}(x)=x^{2}+\mathcal{O}_{m}\left(x^{1+\frac{1}{m}}\right)$, as desired. Thus for a fixed $m \geq 2$, almost all generalized hyperharmonics are not integers.

Proof of Theorem 1.4. We will use the part (2) of Theorem 1.3. Note that we cannot apply this part directly, as the big-O term depends on $m$ as well. So our method to obtain the theorem will be more delicate. Observe that

$$
T(x)=\sum_{1 \leq m \leq x} S_{m}(x) .
$$

Now we split equation (2.10) into three parts, i.e.

$$
T(x)=T_{1}(x)+T_{2}(x)+T_{3}(x),
$$

where

$$
\begin{aligned}
& T_{1}(x)=\sum_{1 \leq m<4} S_{m}(x), \\
& T_{2}(x)=\sum_{4 \leq m<2 \sqrt{x}+1} S_{m}(x), \\
& T_{3}(x)=\sum_{2 \sqrt{x}+1 \leq m \leq x} S_{m}(x) .
\end{aligned}
$$

Clearly

$$
T_{1}(x)=\mathcal{O}\left(x^{2}\right) .
$$

Now we estimate the proper subsums given in (2.12) and (2.13). There exists an absolute constant $n_{0}$ which does not depend on $m$ such that if $n \geq n_{0}$, then

$$
\left(n-n^{0.525}\right)^{m}-n^{0.525}+1 \geq n^{\frac{m}{2}},
$$

if $m \geq 4$. Now consider the following Figure 2 . 


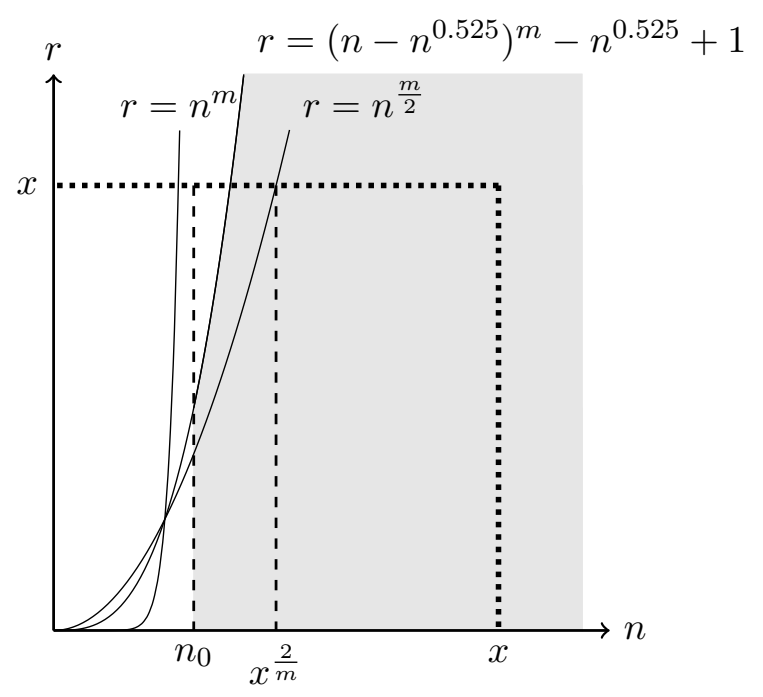

Figure 2. For a fixed $m \geq 4$, the shaded area represents the lattice points $(n, r)$ where the corresponding generalized hyperharmonic number $H_{n}^{(m, r)}$ is not an integer.

By the argument in Theorem 1.3 (2) and equation (2.15), we obtain that

$$
S_{m}(x)=x^{2}+\mathcal{O}\left(x^{1+\frac{2}{m}}\right),
$$

when $m \geq 4$. To estimate the sum $T_{2}(x)$, observe by (2.16) that

$$
\begin{aligned}
T_{2}(x) & =\sum_{4 \leq m<2 \sqrt{x}+1} S_{m}(x)=\sum_{4 \leq m<2 \sqrt{x}+1}\left(x^{2}+\mathcal{O}\left(x^{1+\frac{2}{m}}\right)\right) \\
& =\sum_{4 \leq m<2 \sqrt{x}+1} x^{2}+\mathcal{O}\left(\sum_{4 \leq m<2 \sqrt{x}+1} x^{1+\frac{2}{m}}\right) \\
& =\sum_{4 \leq m<2 \sqrt{x}+1} x^{2}+\mathcal{O}\left(x^{1+\frac{2}{4}} \sum_{4 \leq m<2 \sqrt{x}+1} 1\right) \\
& =\sum_{4 \leq m<2 \sqrt{x}+1} x^{2}+\mathcal{O}\left(x^{\frac{3}{2}} \cdot 2 \sqrt{x}\right)=\sum_{4 \leq m<2 \sqrt{x}+1} x^{2}+\mathcal{O}\left(x^{2}\right)
\end{aligned}
$$

as $m \in[4,2 \sqrt{x}+1]$. For the last sum, note that

$$
\begin{aligned}
T_{3}(x) & =\sum_{2 \sqrt{x}+1 \leq m \leq x} S_{m}(x) \\
& =\sum_{2 \sqrt{x}+1 \leq m \leq x} x^{2}+\mathcal{O}\left(\sum_{2 \sqrt{x}+1 \leq m \leq x} x^{1+\frac{2}{m}}\right) \\
& =\sum_{2 \sqrt{x}+1 \leq m \leq x} x^{2}+\mathcal{O}\left(x \cdot x^{1+\frac{1}{\sqrt{x}}}\right)=\sum_{2 \sqrt{x}+1 \leq m \leq x} x^{2}+\mathcal{O}\left(x^{2}\right),
\end{aligned}
$$

as $x^{\frac{1}{\sqrt{x}}}=e^{\frac{\log x}{\sqrt{x}}}=\mathcal{O}(1)$. Now combining equations (2.14), (2.17) and (2.18) yields to

$$
T(x)=\sum_{m \leq x} x^{2}+\mathcal{O}\left(x^{2}\right)=x^{3}+\mathcal{O}\left(x^{2}\right),
$$

and this concludes the theorem. 
Proof of Theorem 1.5 (1). For the first part, let $\alpha$ be a limit point of the set $\mathcal{H}$. As 1 is in $\mathcal{H}$, we may suppose that $\alpha$ is different from 1 . Thus, there exists a sequence $\left\{h_{n_{k}}^{\left(r_{k}\right)}\right\}$ such that

$$
\lim _{k \rightarrow \infty} h_{n_{k}}^{\left(r_{k}\right)}=\alpha
$$

Since $h_{1}^{(r)}=1$ for any $r$, we can say that $n_{k}>1$ for $k \geq 1$. Assume that the sequence $\left\{n_{k}\right\}$ is unbounded. As $h_{n_{k}}^{\left(r_{k}\right)} \geq h_{n_{k}}$ by the recursion formula (1.7) of hyperharmonics, we deduce that the sequence $\left\{h_{n_{k}}^{\left(r_{k}\right)}\right\}$ is also unbounded since the harmonic series diverges. As a consequence, we see that the sequence $\left\{n_{k}\right\}$ is finite and does not contain 1 . Next, assume that the sequence $\left\{r_{k}\right\}$ is unbounded. By the recursion formula (1.7) of hyperharmonics again, we observe that if $n \geq m$ then $h_{n}^{(r)} \geq h_{m}^{(r)}$. Therefore, we get that

$$
\left\{h_{n_{k}}^{\left(r_{k}\right)}\right\} \geq\left\{h_{2}^{\left(r_{k}\right)}\right\}=\frac{2 r_{k}+1}{2} .
$$

In other words, the sequence $\left\{h_{n_{k}}^{\left(r_{k}\right)}\right\}$ is also unbounded. Thus, the sequence $\left\{r_{k}\right\}$ is finite. Hence $\left\{h_{n_{k}}^{\left(r_{k}\right)}\right\}$ is finite and this yields that $\alpha$ belongs to $\mathcal{H}$.

Proof of Theorem 1.5 (2). Let $\beta$ be a limit point of $\mathcal{G}$. We may assume that $\beta \neq 1$ and $\beta \neq \zeta(k)$ for any $k \geq 2$, as they are the limit points of the set. Write

$$
\lim _{k \rightarrow \infty} H_{n_{k}}^{\left(m_{k}, r_{k}\right)}=\beta .
$$

For $k$ is large enough, we may assume that $n_{k}, r_{k} \geq 2$, because if $r=1$ then the limit is already contained in $\mathcal{G}$, as

$$
\lim _{n \rightarrow \infty} H_{n}^{(m)}=\zeta(m), \quad \lim _{m \rightarrow \infty} H_{n}^{(m)}=1 .
$$

Suppose that $n_{k}$ tends to infinity. By the recursion formula (1.9), we have that

$$
H_{n_{k}}^{\left(m_{k}, r_{k}\right)}=\sum_{i=1}^{n_{k}} H_{i}^{\left(m_{k}, r_{k}-1\right)} \geq n_{k}
$$

as $r_{k} \geq 2$. Therefore, if $n_{k}$ is unbounded, then so is the set $\left\{H_{n_{k}}^{\left(m_{k}, r_{k}\right)}\right\}$. Thus the set $\left\{n_{k} \mid k \geq 1\right\}$ must be finite. Clearly we also have $H_{n_{k}}^{\left(m_{k}, r_{k}\right)} \geq H_{2}^{\left(m_{k}, r_{k}\right)}$. Now suppose that $r_{k}$ tends to infinity, as $k$ tends to infinity. Since

$$
H_{2}^{\left(m_{k}, r_{k}\right)}=H_{1}^{\left(m_{k}, r_{k}-1\right)}+H_{2}^{\left(m_{k}, r_{k}-1\right)},
$$

we see by induction that $H_{2}^{\left(m_{k}, r_{k}\right)} \geq r_{k}$. So again, we get that $\left\{H_{n_{k}}^{\left(m_{k}, r_{k}\right)}\right\}$ is not bounded. Hence $\left\{\left(n_{k}, r_{k}\right) \mid k \geq 1\right\}$ is finite. As a result of this, we may assume that $\left(n_{k}, r_{k}\right)=(n, r)$ for some fixed $n, r \geq 2$. If the set $\left\{m_{k} \mid k \geq 1\right\}$ is also finite, we see that $\beta \in \mathcal{G}$. Now suppose that $m_{k}$ tends to infinity, as $k$ goes to infinity. We know by recursion that

$$
H_{n}^{(m, r)}=H_{1}^{(m, r-1)}+H_{2}^{(m, r-1)}+\cdots+H_{n}^{(m, r-1)} .
$$

By induction on $n+r$, this indicates that $\lim _{m \rightarrow \infty} H_{n}^{(m, r)}$ is a positive integer. Finally by equation (2.19), we get that

$$
\lim _{m \rightarrow \infty} H_{2}^{(m, r)}=r .
$$

This concludes the proof of the second part of Theorem 1.5.

Proof of Theorem 1.5 (3). By the second part of Theorem 1.5, we know that for a fixed $n, r \in \mathbb{N}$, where $n \geq 2$, the limit $\lim _{m \rightarrow \infty} H_{n}^{(m, r)}$ is an integer. But we also know that the sequence $\left\{H_{n}^{(m, r)}\right\}_{m \geq 1}$ is decreasing as $n \geq 2$. Thus the third part of Theorem 1.5 follows. 


\section{Concluding remarks}

As we mentioned earlier, currently we do not have any such fundamental equation like (1.8) for generalized hyperharmonic numbers when $m \geq 2$. Therefore, it is hard to adapt combinatorial and algebraic approaches which were given in [11], since there does not exist any fixed binomial term that we can compute its $p$-adic valuation for a given prime $p$. Moreover, it can be shown by using [16] that there are some generalized hyperharmonic numbers whose 2 -adic valuation is greater than 0 . The following Table 1 contains such examples.

\begin{tabular}{|c|c|c|c|c|}
\hline$n$ & $m$ & $r$ & $H_{n}^{(m, r)}$ & $\nu_{2}\left(H_{n}^{(m, r)}\right)$ \\
\hline 2 & 2 & 2 & $9 / 4$ & -2 \\
\hline 5 & 2 & 3 & $22073 / 1200$ & -4 \\
\hline 5 & 3 & 3 & $1189951 / 72000$ & -6 \\
\hline 5 & 3 & 32 & $119538143 / 2250$ & -1 \\
\hline 7 & 2 & 64 & $270270407641 / 2205$ & 0 \\
\hline 14 & 2 & 11 & $83741315760829 / 61486425$ & 0 \\
\hline 15 & 2 & 11 & $433932992403542 / 184459275$ & 1 \\
\hline 15 & 2 & 12 & $1653452996692253 / 312161850$ & -1 \\
\hline 15 & 2 & 64 & $12505713644625510548024 / 14189175$ & 3 \\
\hline
\end{tabular}

Table 1. The generalized hyperharmonic numbers and their 2-adic valuations for different values of $n, m$ and $r$.

As it can be seen from these values, one cannot give the upper bound 0 for the 2-adic valuation of $H_{n}^{(m, r)}$ unlike the hyperharmonic case which is obtained in [13, Corollary 3.7]. Moreover, it was proved in [11, Theorem 2] that if $n$ is even or $r$ is odd, then the corresponding hyperharmonic number $h_{n}^{(r)}$ is not an integer, as $\nu_{2}\left(h_{n}^{(r)}\right) \leq-1$. However, this does not work for generalized hyperharmonic numbers, as we see from the table above.

\section{References}

[1] E. Alkan, Approximation by special values of harmonic zeta function and log-sine integrals, Commun. Number Theory Phys. (10) 7, 515-550, 2013.

[2] E. Alkan, H. Göral and D.C. Sertbaş, Hyperharmonic Numbers can Rarely be Integers, Integers, 18 Paper No. A43, 1-16, 2018.

[3] T.M. Apostol, Introduction to Analytic Number Theory, Springer-Verlag, New York, First edition, 1976.

[4] T.M. Apostol and T.H. Vu, Dirichlet Series Related to the Riemann Zeta Function, J. Number Theory, 19, 85-102, 1984.

[5] R.C. Baker, G. Harman and J. Pintz, The difference between consecutive primes, II, Proc. Lond. Math. Soc. (3), 83, 532-562, 2001.

[6] B.C. Berndt, Ramanujan's Notebooks, Part V, Springer-Verlag, New York, 1998.

[7] D. Borwein, J.M. Borwein and R. Girgensohn, Explicit evaluation of Euler sums, Proc. Edinb. Math. Soc. (2), 38, 277-294, 1995.

[8] K. Boyadzhiev and A. Dil, Euler sums of hyperharmonic numbers, J. Number Theory, 147, 490-498, 2015.

[9] M. Cenkçi, A. Dil and I. Mező, Evaluation of Euler-like sums via Hurwitz zeta values, Turkish J. Math. 41, 1640-1655, 2017.

[10] J.H. Conway and R.K. Guy, The Book of Numbers, Springer-Verlag, New York, 1996. 
[11] H. Göral and D.C. Sertbaş, Almost all Hyperharmonic Numbers are not Integers, J. Number Theory, 171, 495-526, 2017.

[12] H. Göral and D.C. Sertbaş, A congruence for some generalized harmonic type sums, Int. J. Number Theory, 14 (4), 1033-1046, 2018.

[13] H. Göral and D.C. Sertbaş, Divisibility Properties of Hyperharmonic Numbers, Acta Math. Hungar. 154 (1), 147-186, 2018.

[14] K. Kamano, Dirichlet series associated with hyperharmonic numbers, Mem. Osaka Inst. Tech. Ser. A, 56 (2), 11-15, 2011.

[15] I. Mezö, About the non-integer property of hyperharmonic numbers, Ann. Univ. Sci. Budapest. Sect. Math. 50, 13-20, 2007.

[16] W.A. Stein et. al., Sage Mathematics Software (Version 6.10.rc2), The Sage Development Team, 2015, http://www. sagemath.org.

[17] L. Theisinger, Bemerkung über die harmonische reihe, Monatsh. Math. Phys. 26, 132-134, 1915. 